\title{
Validation of a Model and a Simulator for Road Cycling on Real Tracks
}

\author{
Thorsten Dahmen · Roman Byshko · Dietmar Saupe · Martin Röder · Stephan \\ Mantler
}

\begin{abstract}
In this study, methods for data acquisition, analysis, modelling, and simulation of performance parameters in road cycling on real tracks were developed an evaluated. A simulator was designed to facilitate the measurement in a laboratory environment. The simulation included real height profiles and a video playback that was synchronised with the cyclist's current virtual position on the track and online visualisation of course and performance parameters. Field data obtained on mountain tracks in this study were compared with the state-of-the art mathematical model for road cycling power, established by Martin et al. in 1998, which accounts for the gradient force, air resistance, rolling resistance, frictional losses in wheel bearings, and inertia. The model described the performance parameters accurately with correlation coefficients of $0.96-0.99$ and signal-to-noise ratios of 19.7-23.9 dB. It was shown that the mathematical model can be implemented on an ergometer for simulating rides on real courses providing similar quality measures when comparing field and simulator measurements.
\end{abstract}

Keywords road cycling - performance parameters . mathematical model $\cdot$ simulation $\cdot$ model validation $\cdot$ height profiles

This research was supported by the DFG Research Training Group GRK 1042, "Explorative Analysis and Visualisation of Large Information Spaces".

Thorsten Dahmen, Roman Byshko, Dietmar Saupe

University of Konstanz, Germany.

E-mail: dietmar.saupe@uni-konstanz.de

Martin Röder

Hyperstone GmbH, Konstanz, Germany

Stephan Mantler

VRVis Centre for Virtual Reality and Visualisation Research, Vienna, Austria

\section{Introduction}

Computer science in sports is an emerging interdisciplinary field, which has evolved in the last 20 to 30 years focusing on the following areas of research: data acquisition, processing, and analysis, modelling and simulation, data bases and expert systems, multimedia and presentation, and IT networks/communication. Recording devices for a host of physical and physiological parameters have become available both to professional athletes as well as to non-professional sportsmen. These parameters are used for monitoring and measuring sports activities in the lab, during training, and even in competition. However, given the vast amount and many types of data available, it still remains difficult to efficiently extract the relevant information.

This contribution focuses on road cycling on real tracks and methods to acquire, process, model, and analyse performance data. Furthermore, a simulator is realised and model-, real-world-, and simulation data are compared for validation.

\subsection{Related work}

Mathematical models have been introduced to model road cycling performance, e.g., [1-4]. These models were derived from the law of conservation of energy. The state-of-the art mathematical model for road cycling power, established by Martin et al. in 1998, [2], is based on a differential equation that accounts for the gradient force, air resistance, rolling resistance, frictional losses in wheel bearings, and inertia. In that contribution, the model was validated by performing road cycling trials on an almost flat $(0.3 \%$ slope from construction plans) and straight concrete road with nominal constant speeds. A linear regression between the average power measured by an SRM power meter and the power predicted by the model, given the actual average speed, yielded 
a slope of 1.00 for the regression line, a correlation coefficient of 0.97 and a standard error of measurement of the difference between measured and modelled power of $2.7 \mathrm{~W}$ (average SRM power $172.8 \mathrm{~W}$ ). The variation of the data that the model accounts for was $97 \%$.

The mathematical models were used to predict time trial performance [4], required power output during cycling [2], and to derive optimal pacing strategies for time trials in variable synthetic terrain and wind conditions [5-9]. Moreover, the models were applied in on-road measurements to identify physical parameters like rolling friction and aerodynamic drag [10] and the coefficients of the model equation some of which combine several physical parameters [11].

These mathematical models also provide the means upon which ergometers for road biking simulation must be based. For example, in simulations based on ergometers using an eddy current brake the applied brake force is computed as a function of various mechanical friction parameters, the current speed, and the road slope at the current position.

Although mathematical models for road cycling have been successfully applied in various contexts as outlined above, several issues remain to be studied. The state-of-theart model of Martin et al. was validated only for flat courses and using only average measurement values. Thus, dynamic aspects such as variable speed or power output and variable terrain were not included in the study. This implies that the model equation was tested only with respect for its steadystate solution, rather than as an equation for a dynamical system. Moreover, when applied in such a dynamic context, precision of the numerical solution of the differential equation may be another limiting factor for the applicability of the model.

Regarding road cycling simulators there does not seem to exist a detailed account of how to implement the appropriate model equations together with the respective hardware design. Moreover, the commercial ergometers are primarily intended for training purposes and not for scientific studies. Therefore, validations of such simulators including comparisons with corresponding measurements in the field have not yet been undertaken.

\subsection{Aims and objectives}

This contribution aims at the entire cycle of data acquisition, filtering, analysis, modelling, and prediction of complex sport performance data. Endurance sports are particularly suitable applications since they allow for long-term data series, that can be expected to be more homogeneous, and that depend to a lesser degree on chance events than, e.g., game sports. Currently, the work focuses on road cycling that may later be extended to include, e.g., running and rowing.
This paper focuses on a comparison of performance parameters in the field, on a lab simulator, and in the mathematical model. Previously, validations of mathematical models for cycling performance were carried out by comparing predictions of the models to measurements on a flat course. This study focuses on two uphill tracks with varying steepness. Specifically, the cyclist's climbing progress on real outdoor rides on these tracks together with measurements of a power meter is compared to predictions of the mathematical model for these tracks.

Another contribution of this paper is the comparison between performance parameters measured by the simulator and those calculated by the mathematical model. The main purpose of this task is to provide a means to evaluate the extent to which a lab ergometer ride can accurately simulate an outdoor ride on real-world tracks. Such simulations may then be used by athletes to prepare for competitions on unknown courses.

The following subsection outlines the mathematical models for cycling power that is used for the comparison with field and lab tests. In Section 2 the cycling courses, the equipment, the test setup, the data preprocessing, and the means of comparison of measurements and model prediction are discussed. The last three sections include the results, their discussion, and conclusions.

\subsection{Mathematical models}

Beginning in the 1980s, mathematical models have been developed to describe the relation between pedalling power and speed during road cycling. These models comprise a multitude of physical phenomena, but their accuracy could not be determined before the SRM power meter [12] became available commercially in 1994. Then, in 1998 Martin et al. summarised the significant components to form a mathematical model for road cycling power [2].

The model is based on an equilibrium of resistance power and pedalling power $P_{\text {ped }}$ provided by a cyclist to propel his bicycle. The resistance power is composed of power due to gain in potential energy $P_{\text {pot }}$, aerodynamic drag $P_{\text {air }}$, frictional losses in wheel bearings $P_{\text {bear }}$, rolling friction $P_{\text {roll }}$ and gain in kinetic energy $P_{\text {kin }}$,

$P_{\mathrm{pot}}+P_{\text {air }}+P_{\text {bear }}+P_{\text {roll }}+P_{\text {kin }}=\eta P_{\text {ped }}$.

The efficiency factor $\eta<1$ accounts for frictional loss in the drive chain.

Dividing this equation by the angular speed of the wheels yields the corresponding equilibrium of torques where one has to consider the lever principle using the transmission ratio $\gamma=\frac{n_{\text {front }}}{n_{\text {rear }}}$, i.e., the ratio of the number of teeth on the front sprocket to the number of teeth on the rear 
sprocket:

$T_{\text {pot }}+T_{\text {air }}+T_{\text {bear }}+T_{\text {roll }}+T_{\text {kin }}=\frac{\eta}{\gamma} T_{\text {ped }}$.

The pedal torque is equal to the product of the pedal force and the length of the crank: $T_{\text {ped }}=F_{\text {ped }} l_{c}$. Moreover, Equation (1) is divided by the radius of the wheels, $r_{w}$, in order to obtain the equilibrium of resistance and pedalling force acting at the contact area between the rear wheel and the road:

$F_{\text {pot }}+F_{\text {air }}+F_{\text {bear }}+F_{\text {roll }}+F_{\text {kin }}=\frac{\eta}{\gamma} \frac{l_{c}}{r_{w}} F_{\text {ped }}$.

Eventually, the specific mechanical models can be substituted into each component:

$$
\begin{gathered}
\underbrace{m g \frac{\mathrm{d} h}{\mathrm{~d} x}}_{F_{\mathrm{pot}}}+\underbrace{\frac{1}{2} c_{d} \rho A \dot{x}^{2}}_{F_{\mathrm{air}}}+\underbrace{\left(\beta_{0}+\beta_{1} \dot{x}\right)}_{F_{\text {bear }}}+\underbrace{\mu m g}_{F_{\text {roll }}} \\
+\underbrace{\left(m+\frac{I_{w}}{r_{w}^{2}}\right)}_{F_{\mathrm{kin}}} \ddot{x}=\frac{\eta}{\gamma} \frac{l_{c}}{r_{w}} F_{\text {ped }}
\end{gathered}
$$

Here, $x=x(t)$ is the distance travelled as a function of time $t, \dot{x}=v(t)$ is the speed, and $\ddot{x}=\dot{v}(t)$ is the acceleration. The other physical parameters are listed in Table 1. Ambient wind and aerodynamic drag by rotation of spokes is neglected.

Thus, the sought relation can be rewritten as a nonlinear differential equation of the form

$\gamma \frac{r_{w}}{l_{c}} f(x(t), \dot{x}(t), \ddot{x}(t))=\eta F_{\text {ped }}(t)$,

respectively,

$f(x(t), \dot{x}(t), \ddot{x}(t)) \cdot \dot{x}(t)=\eta P_{\text {ped }}(t)$,

where the discounted pedalling force $\eta F_{\text {ped }}(t)$, respectively the discounted pedalling power $\eta P_{\text {ped }}(t)$, occurs as the driving term for the covered distance $x(t)$, as the independent variable, and $f(x, \dot{x}, \ddot{x})$ denotes the left hand side of Equation (3).

The mathematical model is used in two ways.

1. Given the distance measurements $x(t)$ for the duration of a ride, the corresponding pedalling force $F_{\text {ped }}(t)$, respectively pedalling power $P_{\text {ped }}$, is computed by evaluating $f(x(t), \dot{x}(t), \ddot{x}(t))$ in Equation (4). These values are then compared with the actual power measurements provided by the SRM.

2. Given measured or prescribed pedalling power $P_{\text {ped }}(t)$ for the duration of a ride, Equation (5) is resolved for $x(t)$ numerically (using MATLAB's ode45 function). The derived values for the speed $v(t)=\dot{x}(t)$ are then compared with the actual speed measurements provided by the SRM.
Table 1 Physical parameters of the mathematical model. These parameters originate from measurements or were taken from the literature as follows: $m_{c}, m_{b}$ weighted with scales; $I^{\prime}, l_{c}, c_{w}$ manufacturer information; $L$ measured using bicycle speedometer integrated into Garmin Edge 705 device; $h(x)$ measured (see Section 2.4); $I_{w}$ measured by pendulum experiment, [13]; $\beta_{0}, \beta_{1}, \eta$ from [2]; $c_{d}, A$ average value from

\begin{tabular}{|c|c|c|}
\hline \multicolumn{3}{|c|}{ Cyclist/bicycle/simulator } \\
\hline $\begin{array}{l}\text { mass cyclist } \\
\text { mass bicycle } \\
\text { total mass } \\
\text { simulator inertia } \\
\text { wheel circumference } \\
\text { wheel radius } \\
\text { wheel inertia } \\
\text { cross-sectional area } \\
\text { length of crank } \\
\text { bearing coefficient } \\
\text { bearing coefficient } \\
\text { mechanical gear ratio, bicycle } \\
\text { fixed gear ratio, simulator } \\
\text { simulated gear ratio }\end{array}$ & $\begin{array}{l}m_{c} \\
m_{b} \\
m \\
I^{\prime} \\
c_{w} \\
r_{w} \\
I_{w} \\
A \\
l_{c} \\
\beta_{0} \\
\beta_{1} \\
\gamma \\
\gamma^{\prime} \\
\gamma_{\text {sim }}^{\prime}\end{array}$ & $\begin{array}{l}\text { Tab. } 3 \\
10.6 \mathrm{~kg} \\
m_{b}+m_{c} \\
0.543 \mathrm{kgm}^{2} \\
2105 \mathrm{~mm} \\
(2 \pi)^{-1} c_{w} \\
0.28 \mathrm{kgm}^{2} \\
0.4 \mathrm{~m}^{2} \\
175 \mathrm{~mm}^{2} \\
0.091 \mathrm{~N} \\
0.0087 \mathrm{Ns} / \mathrm{m} \\
39 / 27, \ldots, 50 / 12 \\
50 / 13 \\
39 / 27, \ldots, 50 / 12\end{array}$ \\
\hline \multicolumn{3}{|c|}{ Course/environment } \\
\hline $\begin{array}{l}\text { friction factor } \\
\text { gravity factor } \\
\text { drag coefficient } \\
\text { air density } \\
\text { length } \\
\text { height } \\
\text { chain efficiency }\end{array}$ & $\begin{array}{l}\mu \\
g \\
c_{d} \\
\rho \\
L \\
h(x) \\
\eta\end{array}$ & $\begin{array}{l}0.004 \\
9.81 \mathrm{~m} / \mathrm{s}^{2} \\
0.7 \\
1.2 \mathrm{~kg} / \mathrm{m}^{3} \\
\text { Tab. } 2 \\
\text { Subsec. } 2.1 \\
0.975\end{array}$ \\
\hline
\end{tabular}
[14], $\mu$ standard value from Cyclus 2 ergometer for asphalt road.

The mathematical model for cycling on an ergometer used in this study is slightly different. In the following primed symbols $(\cdot)^{\prime}$ are used to denote ergometer quantities. On an ergometer, the cyclist pedals against the power of the eddy current brake $P_{\text {brake }}^{\prime}$, the power that causes changes of the kinetic energy of the flywheel $P_{\text {kin }}^{\prime}$, and frictional losses in the chain (factor $\eta$ ):

$P_{\text {brake }}^{\prime}+P_{\text {kin }}^{\prime}=\eta P_{\text {ped }}^{\prime}$.

Note that mechanical friction with the simulator is not considered, since the manufacturer assured that this is already accounted for in the internal control of the ergometer brake. In analogy to Equations (1) and (2), the equilibrium in terms of torques yields

$T_{\text {brake }}^{\prime}+T_{\text {kin }}^{\prime}=\frac{\eta}{\gamma^{\prime}} T_{\text {ped }}^{\prime}$,

and in terms of forces

$F_{\text {brake }}^{\prime}+F_{\text {kin }}^{\prime}=\frac{\eta}{\gamma^{\prime}} \frac{l_{c}}{r_{w}} F_{\text {ped }}^{\prime}$.

Again, one can insert the specific mechanical model for the inertial force:

$F_{\text {brake }}^{\prime}+\underbrace{\frac{I^{\prime}}{r_{w}^{2}} \ddot{x}}_{F_{\text {kin }}^{\prime}}=\frac{\eta}{\gamma^{\prime}} \frac{l_{c}}{r_{w}} F_{\text {ped }}^{\prime}$. 
The moment of inertia of the ergometer flywheel $I^{\prime}$ corresponds to a combined inertial mass of cyclist and bicycle of $m_{i}^{\prime}=\frac{I^{\prime}}{r_{w}^{2}} \approx 4.86 \mathrm{~kg}$, which is - as with most ergometers - by far too low.

The simulator that was used in the experiments consists of a Cyclus 2 ergometer and a custom control software. The design of the simulator is based on the following mathematical model for which symbols for the quantities of the simulation are denoted with the additional subscript $(\cdot)_{\text {sim }}^{\prime}$ in order to distinguish them from the physical ergometer quantities. The ergometer allows to impose an arbitrary brake force $F_{\text {brake }}^{\prime}$ (equivalent to a brake torque $T_{\text {brake }}^{\prime}=F_{\text {brake }}^{\prime} r_{w}$ ) with a control software that was developed to simulate real courses. The physical models of (3) were used to compute a simulated brake torque $T_{\text {sim,brake }}^{\prime}$ as the sum of simulated torques due to gain in potential energy $T_{\text {sim,pot }}^{\prime}$, aerodynamic $\operatorname{drag} T_{\text {sim,air }}^{\prime}$, friction in the bearings $T_{\text {sim,bear }}^{\prime}$, and rolling resistance $T_{\text {sim,roll }}^{\prime}$. Furthermore, simulation of arbitrary gears without any mechanical changes was enabled. For this purpose, the factor $\gamma_{\text {sim }}^{\prime}$ was introduced to represent virtual gears and was incorporated into the formula to control the brake torque $T_{\text {brake }}^{\prime}$ of the ergometer. The mechanical gear ratio remained fixed $\left(\gamma^{\prime}=\frac{50}{13}\right)$ at all times.

$$
\begin{aligned}
T_{\text {brake }}^{\prime} & =\frac{\gamma_{\text {sim }}^{\prime}}{\gamma^{\prime}} T_{\text {sim,brake }}^{\prime} \\
& =\frac{\gamma_{\text {sim }}^{\prime}}{\gamma^{\prime}}\left(T_{\text {sim,pot }}^{\prime}+T_{\text {sim,air }}^{\prime}+T_{\text {sim,bear }}^{\prime}+T_{\text {sim,roll }}^{\prime}\right) .
\end{aligned}
$$

This modification required an adjustment of other simulation quantities in order to fulfil the following two desired conditions. Firstly, the power $P_{\text {brake }}^{\prime}$ that is absorbed by the brake should match the simulated power for gain in potential energy $P_{\text {sim,pot }}^{\prime}$, aerodynamic $\operatorname{drag} P_{\text {sim,air }}^{\prime}$, friction in the bearings $P_{\text {bear }}^{\prime}$ and rolling resistance $P_{\text {sim,roll }}^{\prime}$.

$$
\begin{aligned}
P_{\text {brake }}^{\prime} & =T_{\text {brake }}^{\prime} \omega^{\prime} \\
& =P_{\text {sim,pot }}^{\prime}+P_{\text {sim,air }}^{\prime}+P_{\text {bear }}^{\prime}+P_{\text {sim,roll }}^{\prime} \\
& =P_{\text {sim,brake }}^{\prime} \\
& =T_{\text {sim,brake }}^{\prime} \omega_{\text {sim }}^{\prime} .
\end{aligned}
$$

Due to (7), it followed that the angular speed in the simulation $\omega_{\text {sim }}^{\prime}$ had to be defined in terms of the angular speed of the ergometer $\omega^{\prime}$ as

$\omega_{\text {sim }}^{\prime}=\frac{\gamma_{\text {sim }}^{\prime}}{\gamma^{\prime}} \omega^{\prime}$

Secondly, the power that changes the kinetic energy of the ergometer flywheel $P_{\text {kin }}^{\prime}$ should match the power due to changes in kinetic energy in the simulation $P_{\text {sim,kin }}^{\prime}$ :

$$
\begin{aligned}
P_{\text {kin }}^{\prime} & =T_{\text {kin }}^{\prime} \omega^{\prime} \\
& =P_{\text {sim,kin }}^{\prime} \\
& =T_{\text {sim,kin }}^{\prime} \omega_{\text {sim }}^{\prime},
\end{aligned}
$$

where $T_{\text {sim,kin }}^{\prime}$ is the torque responsible for the gain in kinetic energy in the simulation. Together with (9),

$$
T_{\text {sim,kin }}^{\prime}=\frac{\gamma^{\prime}}{\gamma_{\text {sim }}^{\prime}} T_{\text {kin }}^{\prime}
$$

applied.

As a consequence, the moment of inertia in the simulation $I_{\text {sim }}^{\prime}$ differed from the moment of inertia of the ergometer flywheel:

$$
\begin{aligned}
I_{\text {sim }}^{\prime} & =\frac{T_{\text {sim,kin }}^{\prime}}{\dot{\omega}_{\text {sim }}^{\prime}} \\
& =\left(\frac{\gamma^{\prime}}{\gamma_{\text {sim }}^{\prime}}\right)^{2} \underbrace{\frac{T_{\text {kin }}^{\prime}}{\dot{\omega}^{\prime}}}_{I^{\prime}},
\end{aligned}
$$

Clearly, $I_{\text {sim }}^{\prime}$ generally does not match the moment of inertia that corresponds to the mass of a cyclist, a bicycle, and the inertia of the bicycle wheels, which is a shortcoming of the simulation. However, as $\gamma^{\prime}=50 / 13$ was fixed and for most of the time larger than the simulated transmission ratio $\gamma_{\text {sim }}^{\prime}$, for most of the time the moment of inertia was closer to realistic values than the physical moment of inertia of the ergometer flywheel. Another technical advantage of the modification was that the operation of the eddy current brake is improved since the ratio of the torque $T_{\text {brake }}^{\prime}$ to be generated and the angular speed $\omega^{\prime}$ was smaller than the corresponding simulation quantities by a factor of $\left(\gamma_{\text {sim }}^{\prime} / \gamma^{\prime}\right)^{2}$.

Two more physical limitations of the ergometer were partly compensated by the simulation software: Firstly, the maximum torque $T_{\text {brake }}^{\prime}$ which the eddy current brake can generate is an approximately linear function of $\omega^{\prime}$ which was measured by setting a high nominal brake torque, pedalling slowly, and recording the brake torque that was actually generated. During the simulation, whenever a higher than possible torque is needed, the simulation software takes over control and decreases $\gamma_{\text {sim }}^{\prime}$ until the ratio $T_{\text {brake }}^{\prime} / \omega^{\prime}$ is in the valid range again. If necessary, it decreases $\gamma_{\text {sim }}^{\prime}$ even below its minimum of $39 / 27$. While riding the simulator such an event is flagged to the user by a synthetically generated sound and also by a change of colour for the display of the simulated gear ratio $\gamma_{\text {sim }}^{\prime}$.

Secondly, the ergometer has no motor, that could accelerate, e.g., when rolling downhill. Moreover, due to the unavoidable mechanical friction, the interface does not allow a nominal brake torque below values that are equivalent to a pedal force of $50 \mathrm{~N}$. When smaller nominal values are required, the simulation software changes to a model-mode, in which the kinetic quantities, i.e., $\omega_{\text {sim }}^{\prime}$, are computed by the software using the mathematical model and thus are independent of the ergometer quantities. This causes an unavoidable discontinuity of $\omega_{\text {sim }}^{\prime}$ when the nominal brake torque is again high enough to switch to the normal operation, but to 

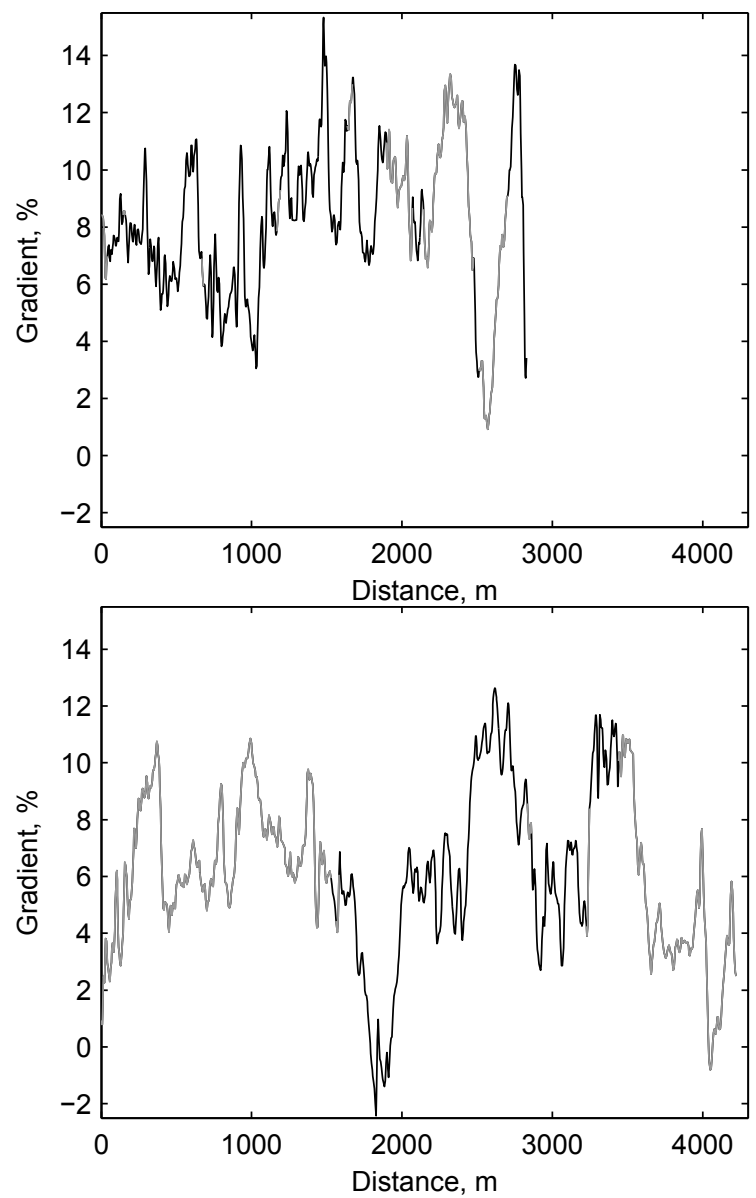

Fig. 1 Gradient $s(x)=\tan (\arcsin (\mathrm{d} h / \mathrm{d} x))$ versus distance of the courses Ottenberg (top) and Wachtbüeler Höhe (bottom). In the sections, where the line is black, a differential GPS signal with a standard deviation of $\sigma_{h}<3 \mathrm{~cm}$ is available and used for the computation of the gradient. In the sections, where the line is grey, the signal reception is degraded due to obstacles like houses and trees and the gradient is estimated using the mathematical model and measurements of power and speed as described in Section 2.1

this extent a simulation of accelerating on a downhill section is possible.

In the following experiments, the courses and pacing strategies were chosen, so that the inertia mismatch and the partly compensated physical ergometer limitations play only a minor role, as will be seen in Section 3.

\section{Methods}

This section provides the details on the experimental setups of the performed bicycle rides as well as on the methods for data acquisition, processing, and analysis of the cycling performance data.

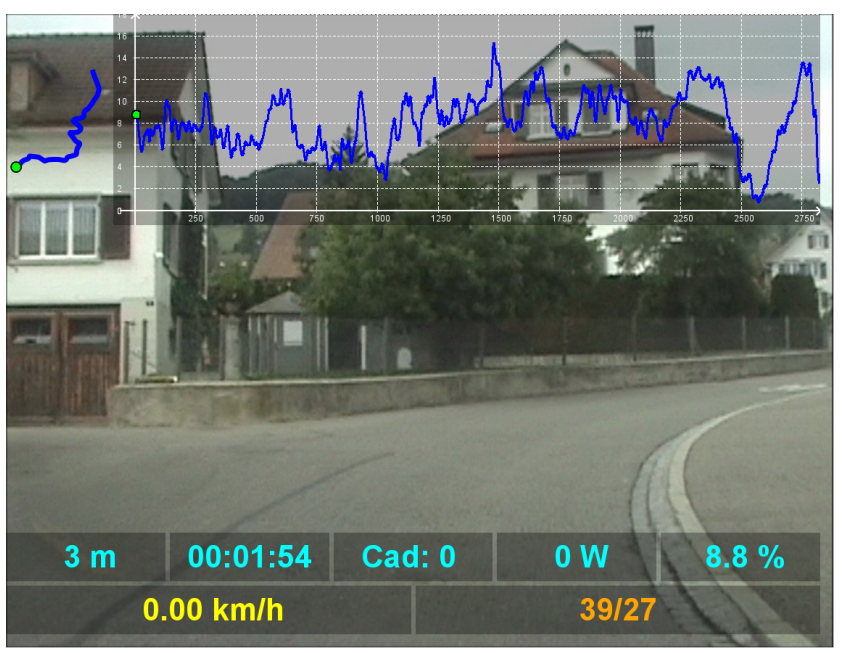

Fig. 2 Screenshot of simulation program window. Scene from the Ottenberg course.

\subsection{Courses}

Two uphill courses of $2.8 \mathrm{~km}$ and $4.2 \mathrm{~km}$ length, with an ascent of about $250 \mathrm{~m}$ each and varying steepness were considered, namely Ottenberg and Wachtbüeler Höhe, located near Weinfelden, Switzerland, and Hüttlingen, Switzerland, respectively. See Table 2 and Figures 1-3 for gradient profiles and picture overviews of the courses. The tracks were recorded by a video camera. The gradient profiles were estimated with a method that combines measurements of a differential GPS device with those of a bicycle computer (Garmin Edge 705) that integrates a speedometer, a standard GPS sensor, and an SRM power meter. The bicycle computer collected measurements of distance, speed, 3dimensional GPS coordinates, and pedalling power during a ride on the track. The details of this method are given in Section 2.4. During field rides the position across the track was kept constant such that the distance to the right edge of the track was approximately $1 \mathrm{~m}$.

\subsection{Equipment}

The cyclists used the same standard road race bicycle (Radon RPS 9.0 with a $60 \mathrm{~cm}$ frame), both in the field and

Table 2 Two courses, called Ottenberg and Wachtbüeler Höhe, were chosen for the tests.

\begin{tabular}{lcc}
\hline & Ottenberg & Wachtbüeler Höhe \\
\hline Length & $2827 \mathrm{~m}$ & $4220 \mathrm{~m}$ \\
Start height & $438 \mathrm{~m}$ & $422 \mathrm{~m}$ \\
End height & $672 \mathrm{~m}$ & $680 \mathrm{~m}$ \\
Average gradient & $8.3 \%$ & $6.1 \%$ \\
Maximum gradient & $15.2 \%$ & $12.6 \%$ \\
Standard deviation of gradient & $2.6 \%$ & $3.0 \%$ \\
\hline
\end{tabular}



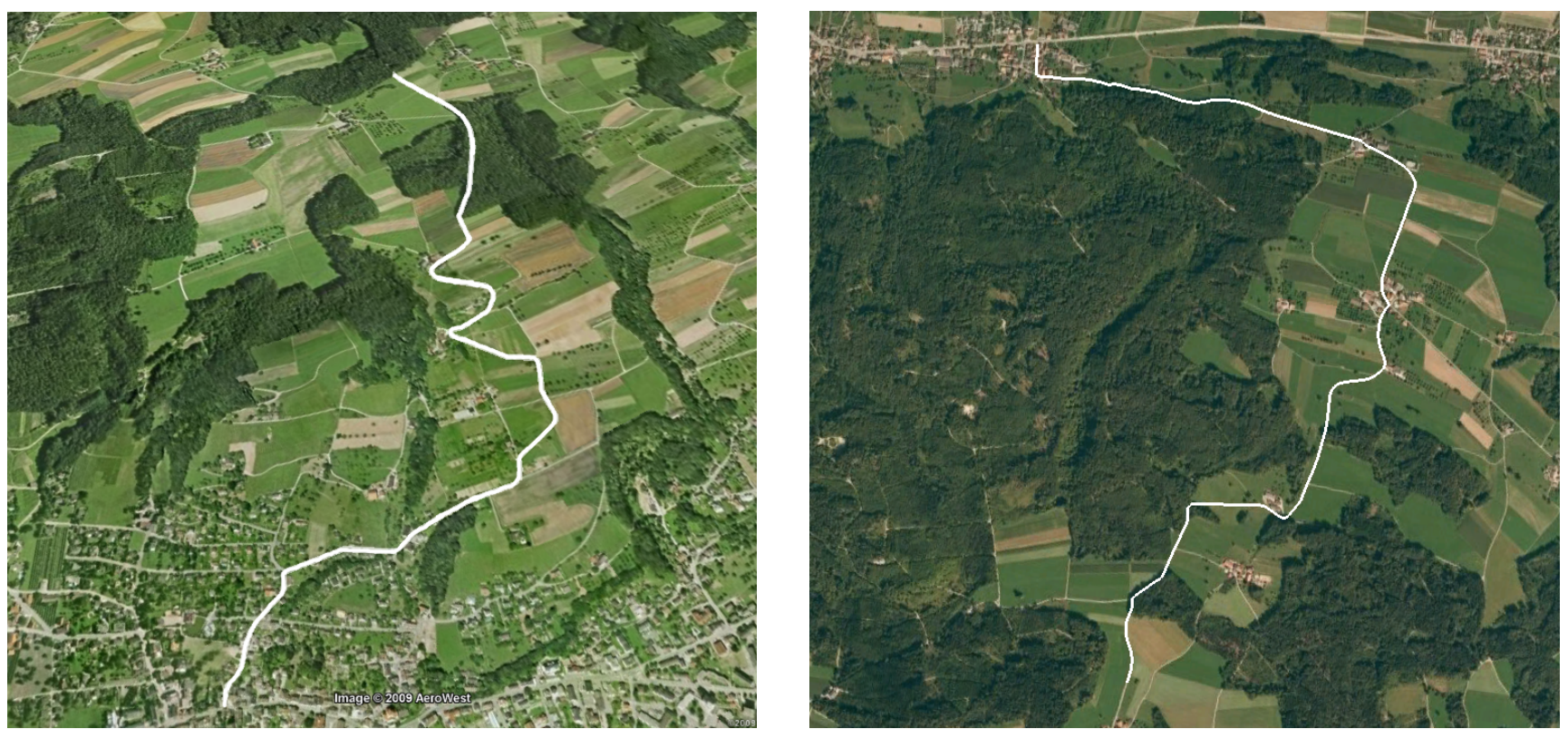

Fig. 3 3D view of the courses: Ottenberg (left), Wachtbüeler Höhe (right).

on the simulator. The bicycle has a 10-speed cassette (13-19 and 21, 23, 27 teeth) and is equipped with a GPS-enabled Garmin Edge 705 bicycle computer which is configured to integrate an SRM power meter with 16 strain gauge strips (Schoberer Rad Messtechnik, Jülich, Germany) attached to the chain wheel (50, 39 teeth). Such devices for measuring power are considered state-of-the-art and have been validated in [2]. The measurements for time, cadence, distance travelled, speed, and power were recorded.

The simulator is based on a Cyclus 2 ergometer [15]. It allows to mount the user's personal bicycle frame and has a flexible front axle attachment which is intended to provide a realistic cycling experience, also when riding out of the saddle. The ergometer can be directly controlled by an external PC-based software at a $2 \mathrm{~Hz}$ rate. In the slave mode the ergometer interface accepts nominal values for the brake torque which is internally transformed into an appropriate braking action of the eddy current brake. An internal sensor measures the rotational speed. Among others, the quantities time and distance counted from ergometry start, cadence, speed, and power can be queried by the interface. The simulator software uses this interface to compute the nominal brake torque according to (7) and the specific mechanical models in (3) and, thus, implements the effects of the actual real-world induced forces.

The simulation includes a video playback that is synchronised with the cyclist's current position on the track and online visualisation of various course and performance parameters, namely the time since the start of the ride, the distance travelled, the current speed, road gradient, cadence, gear ratio, and power output. Moreover, the current position of the cyclist on the course and a plot of the gradient are shown. This visual feedback was displayed during the simulated rides in the lab using an LCD projection unit onto a screen of size of about $1 \mathrm{~m}^{2}$, Figure 2 .

\subsection{Field and simulation tests}

The two selected courses were ridden by several cyclists of differing age, weight, sex, and training level, including novice, occasional, and hobby cyclists as detailed in Table 3. Each ride was performed on the real course as well as with the simulator in the lab. In advance of each ride, the SRM was calibrated according to the manual in order to compensate for the dependency of the sensors on temperature. Since the objective of the experiments was to compare the model predictions for power respectively for speed with the performance on the road and in the lab, the cyclists were instructed to try to maintain either a prescribed constant speed or constant power for each run.

Table 3 Cyclists in the study.

\begin{tabular}{ccccl}
\hline & Age (yrs.) & Weight $(\mathrm{kg})$ & Sex & Experience \\
\hline A & 57 & 72.8 & male & hobby cyclist \\
B & 31 & 74.4 & male & occasional cyclist \\
C & 26 & 83.8 & male & novice cyclist \\
D & 20 & 57.7 & female & occasional cyclist \\
\hline
\end{tabular}


2.4 Data acquisition and processing of outdoor measurements

The following data of the tracks were measured in order to estimate the altitude $\hat{h}(x)$ and the horizontal coordinates, i.e., latitude $\hat{\alpha}(x)$ and longitude $\hat{\beta}(x)$ of the track as functions of the distance $x$. The procedure accounts for the fact that the accuracy of the slope $\mathrm{d} \hat{h} / \mathrm{d} x$ is crucial for a precise modelling and simulation of cycling on a real track.

Measurement 1: An SRM power meter was used in conjunction with a GPS-enabled bicycle computer (Garmin Edge 705) while cycling up the tracks with a moderate pace. The acquired position data, i.e., latitude $\alpha_{\mathrm{gps}}\left(x_{i}\right)$, longitude $\beta_{\mathrm{gps}}\left(x_{i}\right)$, and altitude $h_{\mathrm{gps}}\left(x_{i}\right)$ can be seen as functions of the sample distances $x_{i}$, which were recorded simultaneously by a speedometer. The sampling rate was $1 \mathrm{~Hz}$. The current accuracy of the horizontal coordinates was displayed (3-10 m) on the device, which does not allow to record this information. The accuracy of the altitude is unknown but expected to be worse than the horizontal accuracy. Obstacles like houses or trees along the track degraded the GPS reception, but the signal was never entirely lost. Additionally, the pedalling power $P\left(x_{i}\right)$ was acquired. Overall, measurement 1 provided 3 -dimensional position data with limited precision, distance, and power for the whole track.

Measurement 2: A differential GPS device (Leica GPS900), mounted on the roof of a car, was driven slowly along the tracks. Again, these measurements provided 3dimensional positions given by latitude $\alpha_{\text {dgps }}(j)$, longitude $\beta_{\text {dgps }}(j)$, and altitude $h_{\text {dgps }}(j)$. In addition, standard deviations $\sigma_{\alpha}(j), \sigma_{\beta}(j)$, and $\sigma_{h}(j)$ for each coordinate and sample index $j$ were recorded. Again, the sampling rate was $1 \mathrm{~Hz}$ but there was no distance measurement available. Normally, the standard deviations were in a range of less than a few centimetres. However, in presence of some obstacles, the signal was degraded or even lost (see Figure 1). Overall, measurement 2 provided 3-dimensional positional data with high precision but only for sections of the track where the differential GPS reception was good.

In order to estimate the slope profile of the track these data had to be fused, i.e., the incomplete but precise differential GPS height data of measurement 2 should refine the complete but less precise height data of measurement 1 . First the GPS coordinates $\alpha_{\mathrm{gps}}\left(x_{i}\right)$ and $\beta_{\mathrm{gps}}\left(x_{i}\right)$ were interpolated by means of a 2-dimensional cubic reference spline $\mathbf{s}(x)=(\hat{\alpha}(x), \hat{\beta}(x))$ defining the sought horizontal coordinates as continuous functions of the distance $x$. Then, those points of the differential GPS coordinates $\alpha_{\text {dgps }}(j)$ and $\beta_{\text {dgps }}(j)$ that had high quality, i.e., a standard deviation $\sigma_{h}(j)<3 \mathrm{~cm}$, were projected perpendicularly on the reference spline, so that the distance of the projected point on the spline to the original measurement point was minimal. This yielded points $\mathbf{s}(x(j))=(\hat{\alpha}(x(j)), \hat{\beta}(x(j)))$, where $x(j)$ is the corresponding distance along the spline. The altitudes for these points were obtained from the precise measurements as $\tilde{h}(x(j))=h_{\text {dgps }}(j)$. The altitudes at the spline support points at distances $x_{i}$ which are in the segments with precise differential GPS readings were discarded and replaced by (interpolated) precise measurements $\tilde{h}(x(j))$.

For those track sections where the differential GPS data exceeded the degradation limit or was unavailable, the slope was estimated by resolving the model equation (3) for $\mathrm{d} h / \mathrm{d} x$ and evaluating it at distances $x=x_{j}$ using the measured power $P\left(x_{j}\right)$ and speed $v\left(x_{j}\right)$. However, as the method estimates only the slope rather than the altitude, the slope was integrated, and the resulting altitude function was scaled and shifted by constant factors, so that the resulting altitude $\tilde{h}$ was continuous at the transitions to the sections where differential GPS data was available. A comparison of this method to the slope estimate $\mathrm{d} h_{\mathrm{dgps}} / \mathrm{d} x$ on a section of the track, where precise differential GPS data were available, showed that this slope estimation is more accurate than the slope estimate $\mathrm{d} h_{\mathrm{gps}} / \mathrm{d} x$ obtained by differentiating the standard GPS altitude data.

Finally, $\hat{h}(x)$ was computed as a smoothed cubic spline using the function csaps of Matlab's Curve Fitting Toolbox. It minimises the functional

$J=\sum_{j} p w\left(\sigma_{h}(j)\right)\left|\tilde{h}\left(x_{j}\right)-\hat{h}\left(x_{j}\right)\right|^{2}+(1-p) \int\left|\frac{\mathrm{d}^{2} \hat{h}}{\mathrm{~d} x^{2}}\right|^{2} \mathrm{~d} x$

where, $w\left(\sigma_{h}(j)\right)$ are weights that depend on the standard deviation $\sigma_{h}$ (in meters) at the sample $j$ and $p=0.05$ is a regularisation parameter. The weights were chosen $w\left(\sigma_{h}(j)\right)=$ $\left(1-\sigma_{h}(j)\right)^{10}$ on sections with differential GPS available. On sections without differential GPS reception, the average standard deviation of $\sigma_{h}(j)$ on the sections with differential GPS reception was used. These parameters control the contradictory demands of $\hat{h}(x)$ being both smooth and close to the measured data and were chosen after qualitatively and visually inspecting the result.

Furthermore, a video was taken during another car ride with simultaneous GPS measurements using the Garmin Edge 705 again at a sampling rate of $1 \mathrm{~Hz}$. This allowed to geo-reference each individual video frame. Likewise, for an arbitrary position on the track with a given distance from the starting point one may calculate a corresponding (fractional) video frame number for display in the simulation setting [16].

In order to compare the measured power or speed during an uphill ride with the corresponding model prediction each sample point of the ride had to be assigned the corresponding slope of the track. The direct method would be to evaluate and differentiate the altitude function $\hat{h}$ at the distance $x_{k}$ that was measured at the $k$-th sample point. However, the distances measured with different rides on the same track differ slightly from each other and from those in the height 
profiles because cyclists cannot ride exactly along the same path so that there is a drift error with distance measurements. For this reason, the horizontal coordinates of the GPS measurements of each data point during a ride were projected perpendicularly on the reference spline $\mathbf{s}(x)$ which defines the distance $x$ of the data point that replaces the distance measurement of the ride. The corresponding slope is then defined as $\mathrm{d} \hat{h} / \mathrm{d} x$ evaluated at the obtained distance $x$.

\subsection{Comparison of measurements with model prediction}

The pre-processing of the data measured in the field provided time series of vectors with the components: time $t$, distance $x(t)$, speed $\dot{x}(t)$, power $P_{\text {ped }}(t)$, and gradient $s(x(t))$. For evaluating the rides with constant speed, these values (except $P_{\text {ped }}(t)$ ), inserted in the left side of the model equation (5), yield the predicted power for comparison with the measured power. Section 3 provides the corresponding graphs and gives correlation coefficients and signal-to-noise ratios (SNR). The latter are defined as

$\mathrm{SNR}=10 \log _{10} \frac{\mathrm{MSP}_{\mathrm{ped}}}{\mathrm{MSE}} \mathrm{dB}$

where MSP and MSE denote the mean square power amplitude and the mean square error between the prediction and the actual power, respectively, measured in decibel. The percentage $p$ of the variation of the data that is accounted for by the model is defined by

$p=100\left(1-\frac{\mathrm{MSE}}{\mathrm{MSP}_{\mathrm{ped}}}\right) \%$.

For evaluating the rides with approximately constant power the roles of speed and power in the above are reversed and the SNR and percentage $p$ is defined accordingly.

The analysis for the data obtained with the simulator proceeds similarly. Since the measured speed and power data were automatically linked to the used height profiles no pre-processing to align gradients to the measurements was required.

\subsection{Comparison of simulated rides with outdoor rides}

Consider a comparison of the field rides with constant speed with corresponding simulations in the lab. A direct comparison of measured power values is not appropriate because the difference between power/speed values in the field and in the lab must be attributed to a combination of two effects. Firstly, there is a difference due to model prediction errors and simulator precision. This is the desired quantity to be calculated. Secondly, there is a difference in the actual speed/power that cannot be kept perfectly equal. However, that part is irrelevant for this study. Thus, a normalisation procedure was required that aligns the rides such that the second source of errors was eliminated. This procedure for preprocessing the data was carried out in two steps.

1. Again, the distances of the outdoor rides data points were replaced by the distance parameter of their perpendicular point on the reference spline using the horizontal GPS coordinates.

2. For comparison of power output the speed measures in the field and of the simulator should be identical, but naturally they differed. The mathematical model was used to estimate the power output both in the field and in the lab assuming the preset constant speeds, e.g., $v^{\star}=10$ $\mathrm{km} / \mathrm{h}$.

The compensation for variable speed proceeds as follows. At each point in time the model for the field resp. the simulator predicts a pedalling power $P_{\text {ped }}^{m}=P_{\text {ped }}^{m}(x(t), \dot{x}(t), \ddot{x}(t))$. For constant prescribed speed $v^{\star}$, however, the model would predict at that same location of the course a value of $P_{\text {ped }}^{\star}=$ $P_{\text {ped }}^{m}\left(v^{\star} t, v^{\star}, 0\right)$. The measured power was compensated for the mismatch of the speed simply by multiplying with the factor $P_{\text {ped }}^{\star} / P_{\text {ped }}^{m}$. The compensation must be applied to the measured data from both the field and the simulation. The resulting time series are denoted the normalised power sequences. The procedure for rides with attempted constant power is likewise, normalising the speed to exactly constant power. The resulting normalised power resp. speed was then compared using the same methods as described in the last subsection.

\section{Results}

Table 4 provides a comparison of the contributions to the various model components of the overall work required to perform the ride of cyclist A at approximately constant speed $\left(v^{\star}=10 \mathrm{~km} / \mathrm{h}\right)$ on the Ottenberg. These include the average powers that account for gain in potential energy $\bar{P}_{\text {pot }}$, aerodynamic drag $\bar{P}_{\text {air }}$, frictional losses in wheel bearings $\bar{P}_{\text {bear }}$, rolling friction $\bar{P}_{\text {roll }}$, and gain in kinetic energy $\bar{P}_{\text {kin }}$. The average power was $207.4 \mathrm{~W}$ and the total work was $57.6 \mathrm{Wh}$. It is clear that the fraction due to overcoming the potential energy $\bar{P}_{\text {pot }}$ is dominant while the others are small $\left(\bar{P}_{\text {air }}, \bar{P}_{\text {roll }}\right)$ or even negligible $\left(\bar{P}_{\text {bear }}, \bar{P}_{\text {kin }}\right)$.

The main results are presented by four representative bicycle rides that cover different subjects, courses, and pacing strategies both outdoors on a real course and indoors on the simulator by the same cyclists, using the same pacings. Two of these selected rides were performed with approximately constant speed $v^{\star}$ and power was computed using the model. For the third and fourth one, having approximately constant power $P^{\star}$, the speed was computed. In each case the computed quantity is plotted against the measurement data. The left plots in Figures 4, 5, and 8 show field measurements and 
a) Ottenberg, Cyclist $A, v^{*}=10 \mathrm{~km} / \mathrm{h}$
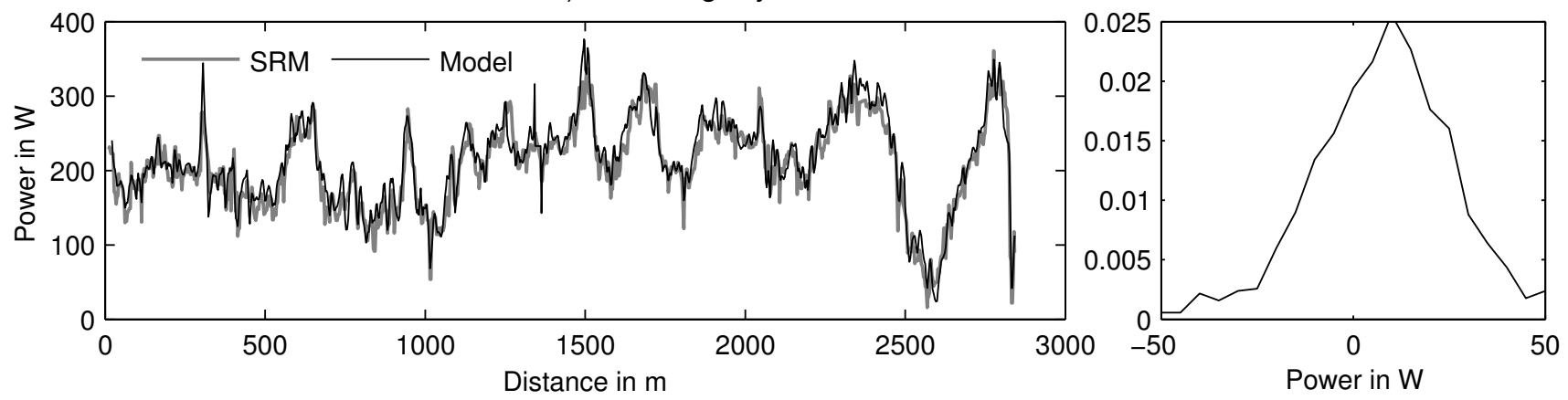

b) Wachtbüeler Höhe, Cyclist B, $v^{*}=11 \mathrm{~km} / \mathrm{h}$
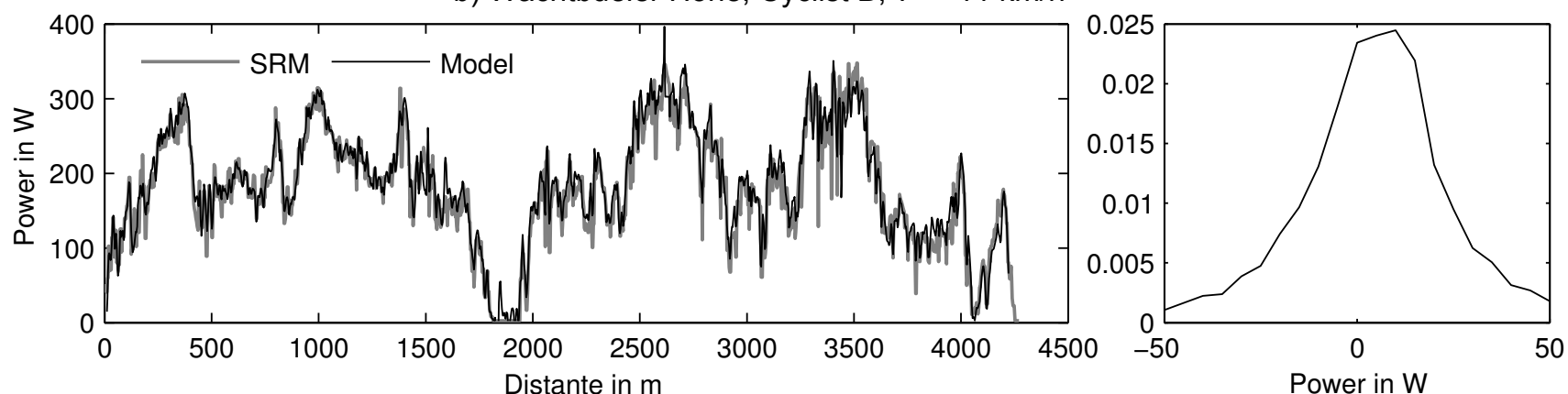

c) Ottenberg, Cyclist C, $P^{*}=160 \mathrm{~W}$
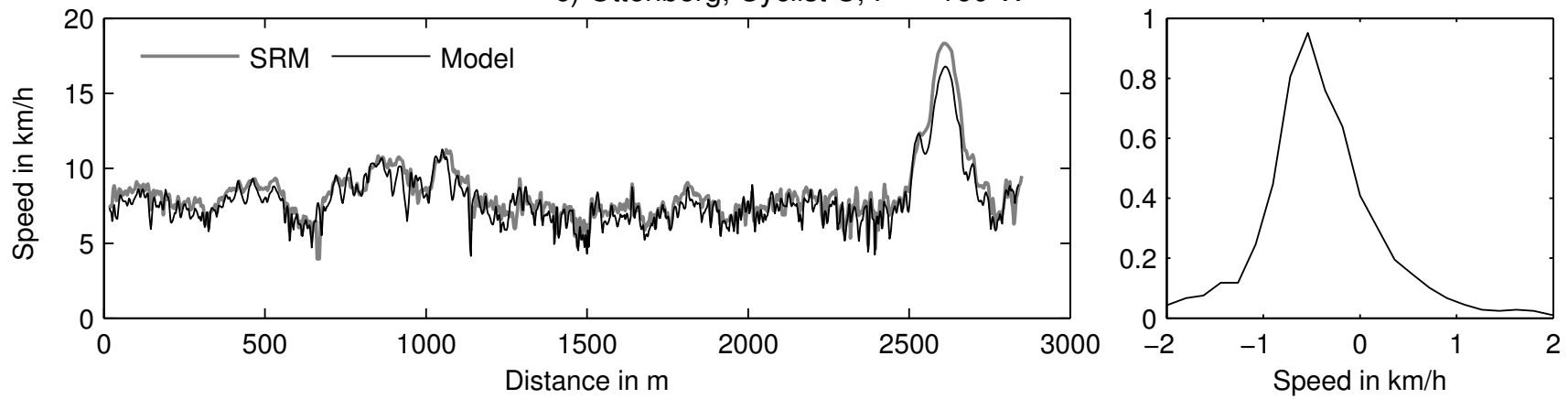

d) Wachtbüeler Höhe, Cyclist D, $P^{*}=150 \mathrm{~W}$
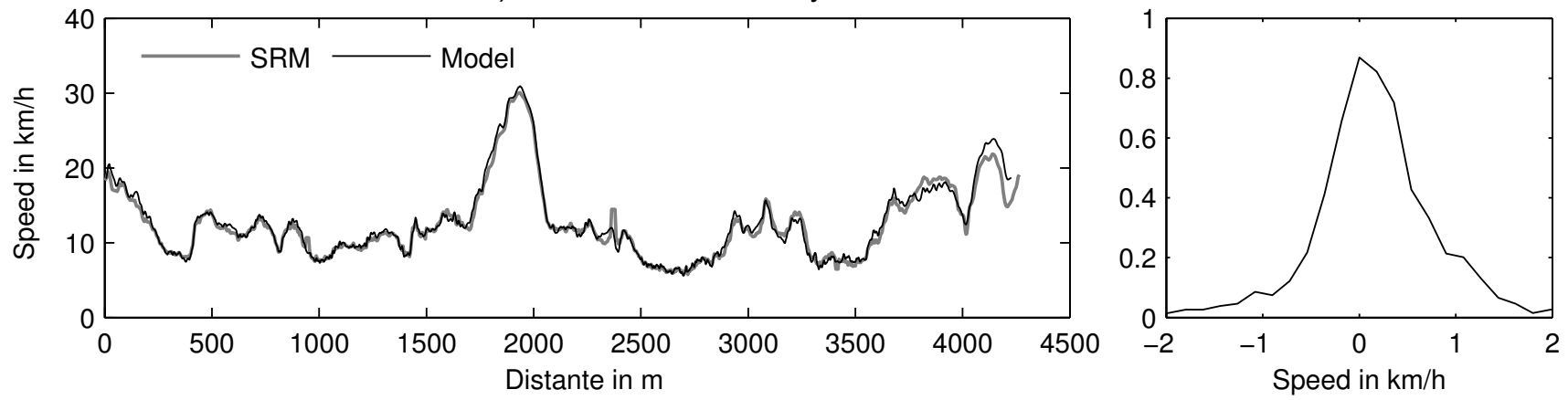

\begin{tabular}{lclccccc}
\hline Cyclist & Condition & Course & $\rho$ & $m_{e}$ & $\sigma_{e}$ & SNR & $p$ \\
\hline A & $v^{\star}=10 \mathrm{~km} / \mathrm{h}$ & Ottenberg & 0.97 & $8.0 \mathrm{~W}$ & $19.1 \mathrm{~W}$ & $20.6 \mathrm{~dB}$ & $99.1 \%$ \\
B & $v^{\star}=11 \mathrm{~km} / \mathrm{h}$ & Wachtbüeler Höhe & 0.98 & $4.9 \mathrm{~W}$ & $20.0 \mathrm{~W}$ & $19.7 \mathrm{~dB}$ & $98.9 \%$ \\
C & $P^{\star}=160 \mathrm{~W}$ & Ottenberg & 0.96 & $-0.41 \mathrm{~km} / \mathrm{h}$ & $0.65 \mathrm{~km} / \mathrm{h}$ & $19.9 \mathrm{~dB}$ & $99.0 \%$ \\
D & $P^{\star}=150 \mathrm{~W}$ & Wachtbüeler Höhe & 0.99 & $0.17 \mathrm{~km} / \mathrm{h}$ & $0.73 \mathrm{~km} / \mathrm{h}$ & $23.9 \mathrm{~dB}$ & $99.6 \%$ \\
\hline
\end{tabular}

Fig. 4 Field rides versus model predictions. In the left plots the grey lines give the power resp. speed SRM measurements. The solid lines show the corresponding predictions of the model. The right plots show the distribution of the prediction error signal, defined as the predicted power resp. speed minus the measured power resp. speed. The prediction errors are analysed in the table. $\rho$ is the correlation coefficient, $m_{e}$ is the mean prediction error, $\sigma_{e}$ is the standard deviation of the prediction error, SNR is the signal-to-noise ratio, and $p$ the percentage of variation explained by the model. Note that in the first two lines power is compared, while in the last two lines speed is compared. 
a) Ottenberg, Cyclist $A, v^{*}=10 \mathrm{~km} / \mathrm{h}$
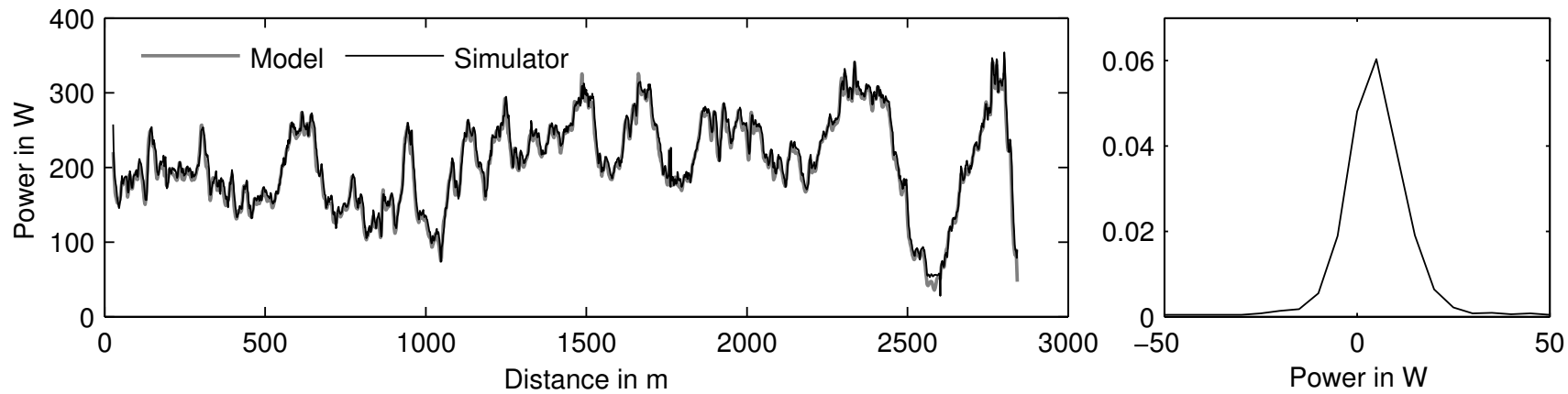

b) Wachtbüeler Höhe, Cyclist $B, v^{*}=11 \mathrm{~km} / \mathrm{h}$
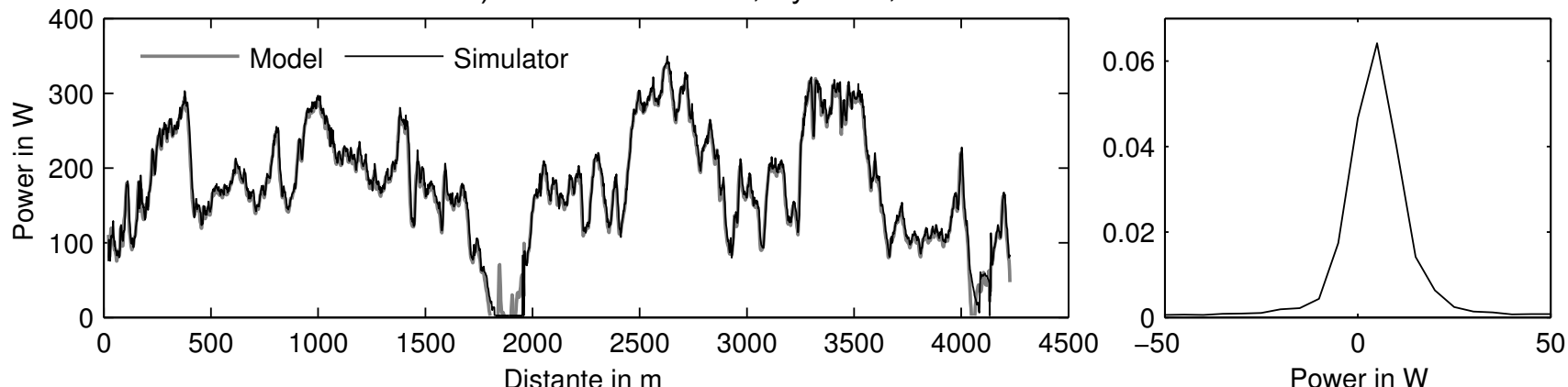

c) Ottenberg, Cyclist $C, P^{*}=160 \mathrm{~W}$
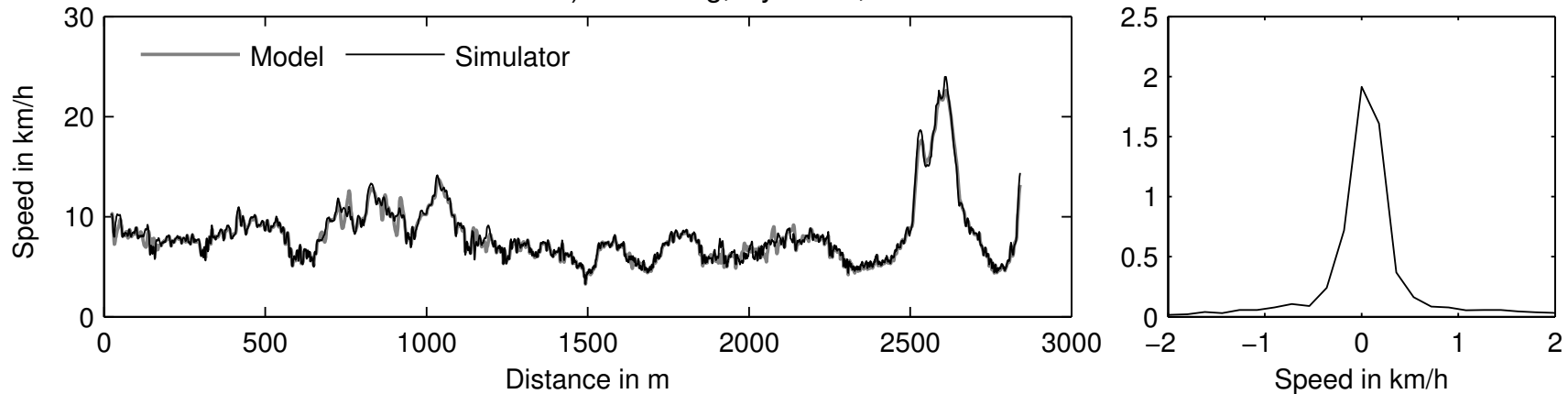

d) Wachtbüeler Höhe, Cyclist $D, P^{*}=150 \mathrm{~W}$
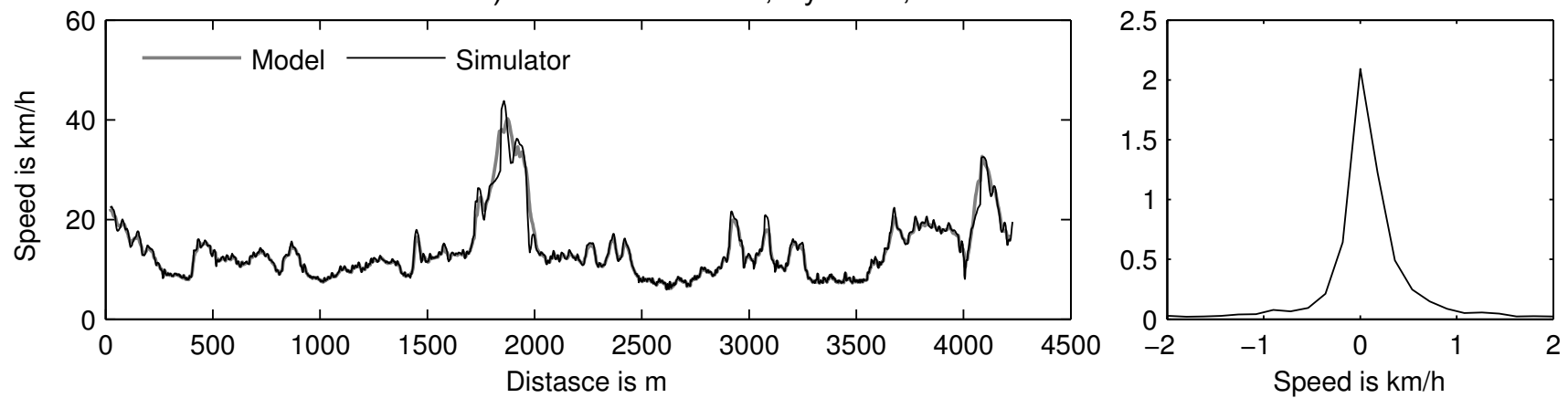

\begin{tabular}{lclccccc}
\hline Cyclist & Condition & Course & $\rho$ & $m_{e}$ & $\sigma_{e}$ & SNR & $p$ \\
\hline A & $v^{\star}=10 \mathrm{~km} / \mathrm{h}$ & Ottenberg & 0.99 & $4.9 \mathrm{~W}$ & $7.5 \mathrm{~W}$ & $27.4 \mathrm{~dB}$ & $99.8 \%$ \\
B & $v^{\star}=11 \mathrm{~km} / \mathrm{h}$ & Wachtbüeler Höhe & 0.99 & $4.7 \mathrm{~W}$ & $9.3 \mathrm{~W}$ & $25.1 \mathrm{~dB}$ & $99.7 \%$ \\
C & $P^{\star}=150 \mathrm{~W}$ & Ottenberg & 0.99 & $0.06 \mathrm{~km} / \mathrm{h}$ & $0.41 \mathrm{~km} / \mathrm{h}$ & $25.2 \mathrm{~dB}$ & $99.7 \%$ \\
D & $P^{\star}=160 \mathrm{~W}$ & Wachtbüeler Höhe & 0.99 & $0.02 \mathrm{~km} / \mathrm{h}$ & $0.87 \mathrm{~km} / \mathrm{h}$ & $23.2 \mathrm{~dB}$ & $99.5 \%$ \\
\hline
\end{tabular}

Fig. 5 Model versus simulated rides. In the left plots the grey lines give the power resp. speed prediction of the model. The solid lines show the measurements of the simulator. The right plots show the distribution of the error signal, defined as the simulator power resp. speed minus the modelled power resp. speed. The errors are analysed in the table. $\rho$ is the correlation coefficient, $m_{e}$ is the mean prediction error, $\sigma_{e}$ is the standard deviation of the prediction error, SNR is the signal-to-noise ratio, and $p$ the percentage of variation explained by the model. (Since starting with $v=0$ causes large errors during the acceleration phase at the beginning, the first $4 \mathrm{~s}$ of the data were cut off and ignored.) 

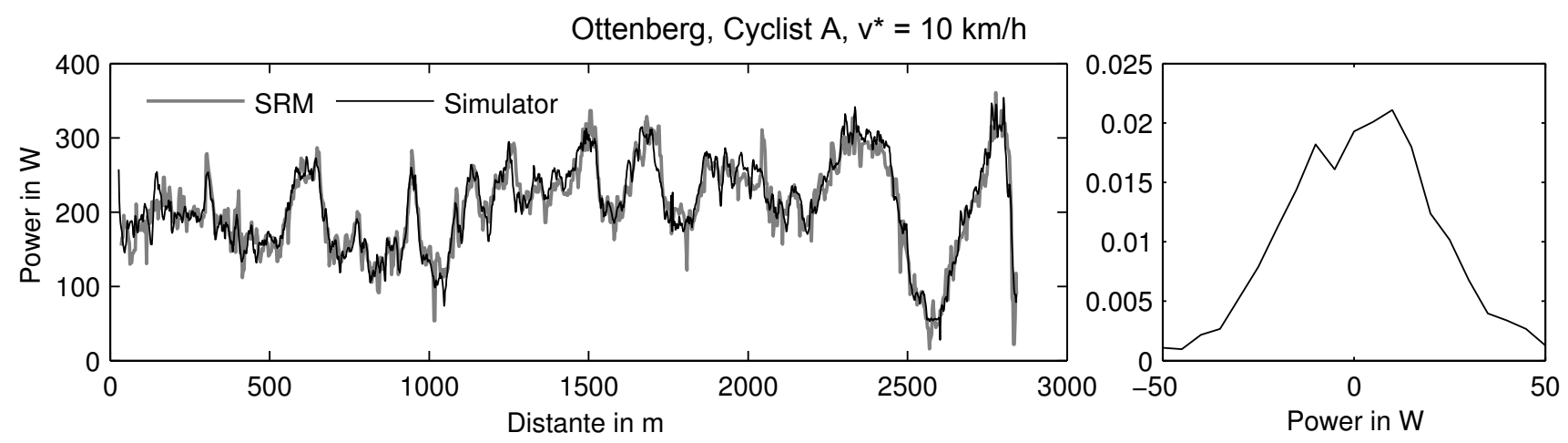

\begin{tabular}{lclccccc}
\hline Cyclist & Condition & Course & $\rho$ & $m_{e}$ & $\sigma_{e}$ & SNR & $p$ \\
\hline A & $v^{\star}=10 \mathrm{~km} / \mathrm{h}$ & Ottenberg & 0.96 & $1.5 \mathrm{~W}$ & $22.4 \mathrm{~W}$ & $19.6 \mathrm{~dB}$ & $98.9 \%$ \\
B & $v^{\star}=11 \mathrm{~km} / \mathrm{h}$ & Wachtbüeler Höhe & 0.97 & $-2.0 \mathrm{~W}$ & $23.5 \mathrm{~W}$ & $18.2 \mathrm{~dB}$ & $98.5 \%$ \\
C & $P^{\star}=160 \mathrm{~W}$ & Ottenberg & 0.90 & $-0.54 \mathrm{~km} / \mathrm{h}$ & $1.41 \mathrm{~km} / \mathrm{h}$ & $14.1 \mathrm{~dB}$ & $96.1 \%$ \\
D & $P^{\star}=150 \mathrm{~W}$ & Wachtbüeler Höhe & 0.94 & $0.67 \mathrm{~km} / \mathrm{h}$ & $2.21 \mathrm{~km} / \mathrm{h}$ & $14.6 \mathrm{~dB}$ & $96.6 \%$ \\
\hline
\end{tabular}

Fig. 6 Field versus simulator ride of cyclist A. In the left plot the grey line gives the power measured by the SRM. The solid line shows the measurement of the simulator. The right plot shows the distribution of the error signal, defined as the simulator power minus the SRM power. The errors are analysed in the table for all rides. $\rho$ is the correlation coefficient, $m_{e}$ is the mean error, $\sigma_{e}$ is the standard deviation, SNR is the signal-to-noise ratio, and $p$ the percentage of variation explained by the model. (Since on the simulator starting with $v=0$ causes large errors during the acceleration phase at the beginning, the first $4 \mathrm{~s}$ of the data were cut off and ignored.)
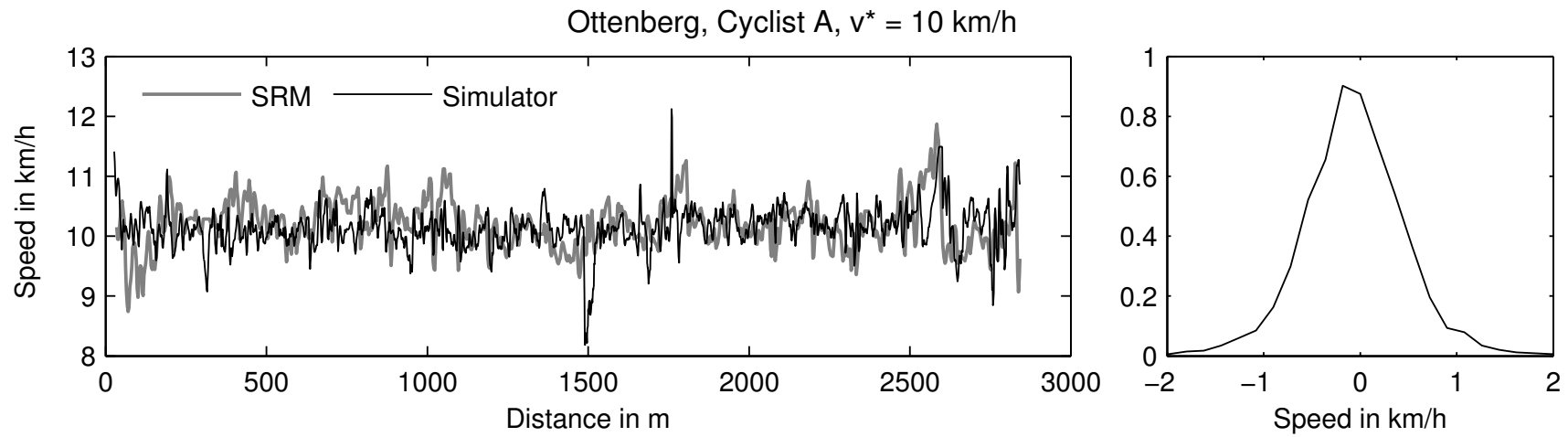

Fig. 7 Field versus simulator ride of cyclist A. The left plot shows the actual speed when the cyclist tried to maintain a constant speed of $10 \mathrm{~km} / \mathrm{h}$ both in the field and on the simulator. The right plot shows the distribution of the difference signal, defined as speed on the simulator minus speed in the field. (Since on the simulator starting with $v=0$ causes large errors during the acceleration phase at the beginning, the first $4 \mathrm{~s}$ of the data were cut off and ignored.)

Table 4 Distribution of power for a ride up the Ottenberg $(2.827 \mathrm{~km}$, $234 \mathrm{~m}$ altitude) at $10 \mathrm{~km} / \mathrm{h}$ requiring a total time of $16 \mathrm{~min} 39 \mathrm{~s}$ and a total energy of $57.6 \mathrm{Wh}$.

\begin{tabular}{lrr}
\hline Power & Average power & Percentage \\
\hline $\bar{P}_{\text {pot }}$ & $190.4 \mathrm{~W}$ & $91.8 \%$ \\
$\bar{P}_{\text {air }}$ & $7.5 \mathrm{~W}$ & $3.6 \%$ \\
$\bar{P}_{\text {bear }}$ & $0.3 \mathrm{~W}$ & $0.1 \%$ \\
$\bar{P}_{\text {roll }}$ & $9.2 \mathrm{~W}$ & $4.4 \%$ \\
$\bar{P}_{\text {kin }}$ & $0.0 \mathrm{~W}$ & $0.0 \%$ \\
\hline total & $207.4 \mathrm{~W}$ & $100.0 \%$ \\
\hline
\end{tabular}

model predictions, simulator measurements and model predictions, and normalised field and simulator measurements, respectively. The intermediate Figures 6 and 7 compare simulator and field measurements before the normalisation had been performed, thus containing an error that is induced by the inability of the cyclists to maintain exactly the same pacing indoors and outdoors. The right plots in each figure show density functions of the distributions of the differences between these pairs of signals. The tables at the bottom in Figures 4-6, and 8 characterise the deviations of the model predictions and measurements by giving the correlation coefficient $\rho$, the mean error $m_{e}$, the standard deviation of the error $\sigma_{e}$, the signal-to-noise ratio SNR as defined in (12), and the percentage $p$ of the variation of the data accounted for by the model given by (13). 
a) Ottenberg, Cyclist $A, v^{*}=10 \mathrm{~km} / \mathrm{h}$

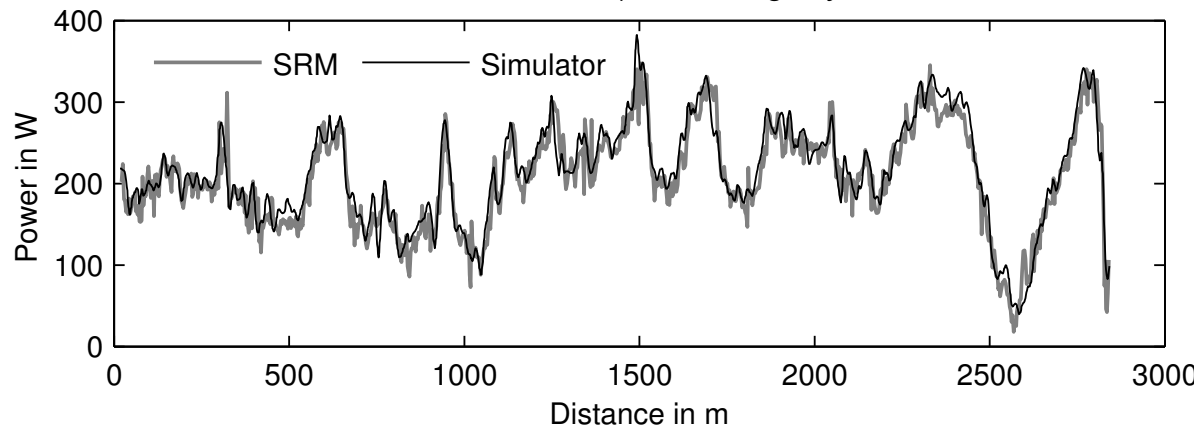

b) Wachtbüeler Höhe, Cyclist B, $v^{*}=11 \mathrm{~km} / \mathrm{h}$

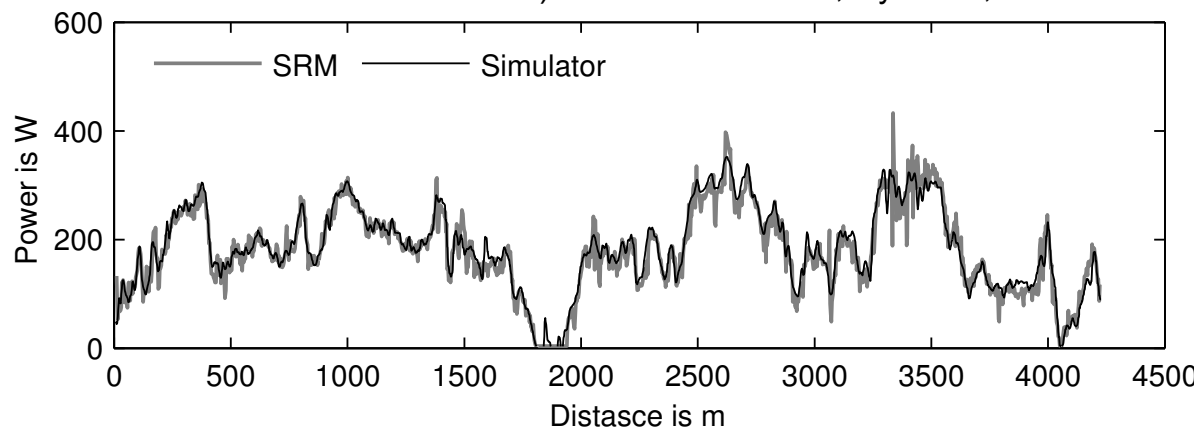

c) Ottenberg, Cyclist C, $P^{\star}=160 \mathrm{~W}$

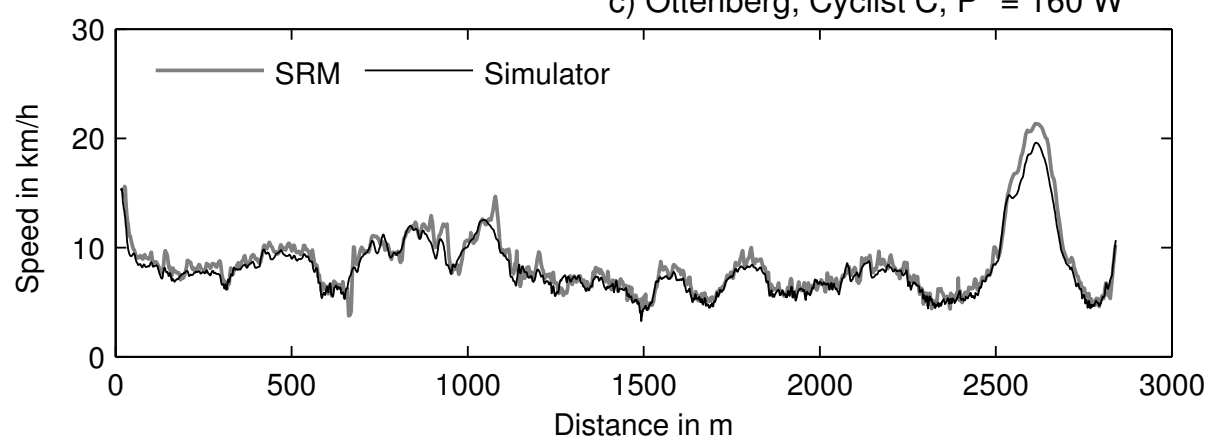

d) Wachtbüeler Höhe, Cyclist D, $P^{*}=150 \mathrm{~W}$
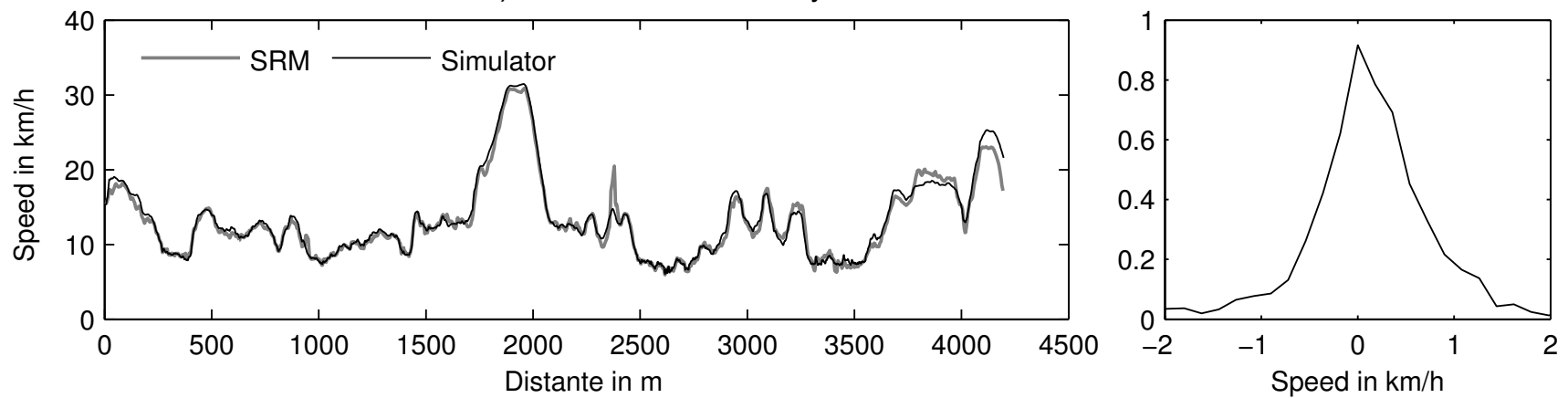

\begin{tabular}{lclccccc}
\hline Cyclist & Condition & Course & $\rho$ & $m_{e}$ & $\sigma_{e}$ & SNR & $p$ \\
\hline A & $v^{\star}=10 \mathrm{~km} / \mathrm{h}$ & Ottenberg & 0.98 & $7.5 \mathrm{~W}$ & $18.5 \mathrm{~W}$ & $20.9 \mathrm{~dB}$ & $99.2 \%$ \\
B & $v^{\star}=11 \mathrm{~km} / \mathrm{h}$ & Wachtbüeler Höhe & 0.98 & $4.8 \mathrm{~W}$ & $19.0 \mathrm{~W}$ & $20.1 \mathrm{~dB}$ & $99.0 \%$ \\
C & $P^{\star}=160 \mathrm{~W}$ & Ottenberg & 0.98 & $-0.45 \mathrm{~km} / \mathrm{h}$ & $0.66 \mathrm{~km} / \mathrm{h}$ & $19.5 \mathrm{~dB}$ & $98.3 \%$ \\
D & $P^{\star}=150 \mathrm{~W}$ & Wachtbüeler Höhe & 0.99 & $0.12 \mathrm{~km} / \mathrm{h}$ & $0.72 \mathrm{~km} / \mathrm{h}$ & $24.6 \mathrm{~dB}$ & $99.7 \%$ \\
\hline
\end{tabular}

Fig. 8 Field versus simulator rides (for normalised measurements). In the left plots the grey lines give the power resp. speed measured by the SRM. The solid lines show the measurements of the simulator. The right plots show the distributions of the error signals, defined as the simulator power minus the SRM power. The errors are analysed in the table. $\rho$ is the correlation coefficient, $m_{e}$ is the mean error, $\sigma_{e}$ is the standard deviation, SNR is the signal-to-noise ratio, and $p$ the percentage of variation explained by the model. (Since on the simulator starting with $v=0$ causes large errors during the acceleration phase at the beginning, the first $4 \mathrm{~s}$ of the data were cut off and ignored.) 


\section{Discussion}

The discussion is organised in three parts: the comparison of the model predictions with the measurements in the field, with those in the lab, and the comparison of the performance in the field with that in the lab.

4.1 Comparison of measurements in the field with model predictions

The results in Figure 4 show that the mathematical model describes the dynamics of power output on an uphill course with good precision. The signal-to-noise ratio was 19.7$23.9 \mathrm{~dB}$ and 98.9 to $99.6 \%$ of the variation of the measured power over the course was accounted for by the model. This finding is even better than the $97 \%$ reported in the previous study [2] of Martin et al. This may be due to the fact that in the previous study the model was evaluated only for the constant prescribed speed (on a flat course) while in this study the model equations were evaluated for all of the time steps, thus, also accounting for variations in speed.

The density functions of the power and speed errors show a hat-shaped form which can be well characterised by the mean values $m_{e}$ and the standard deviations $\sigma_{e}$. Yet, outliers due to systematic errors which occured occasionally, slightly exceed the standard deviation one might expect from the visual impression of the density functions.

For the first ride, $m_{e}$ and $\sigma_{e}$ made up $4 \%$ and $9 \%$ of the average power given in Table 4 . The mean error of the predictions may be due to several factors: the physical model parameters could have been not sufficiently precise. Some were taken from the literature and should be adapted for the bicycle, the courses, and the cyclists. Others were measured with errors. The manufacturer of the SRM power meter claims an accuracy of $\pm 0.5 \%$ for the power measurements, although it was confirmed on personal request that the accuracy has not been validated scientifically. Together with the unknown measurement error of the speedometer this could explain part of the error. As gravity was responsible for the major part of the overall resistance, a small measurement error of the mass could introduce a significant bias, too. For the first ride, the observed bias of $8.0 \mathrm{~W}$ would be compensated for, if the mass of the rider was assumed to be $69.5 \mathrm{~kg}$ instead of $72.8 \mathrm{~kg}$. Although this is too much of a difference to attribute the mean error solely to the inexactness of the used commercial scales, the sum of all propagated measurement errors can be expected to produce mean errors in the range of the observed values.

Besides the measurement error of the power meter resp. the speedometer, it can be assumed that the main source of the standard deviation of the model prediction error was due to errors in the measurement of the road gradient. These errors in the slope measurement are due to a combination of errors of the distance measurement, the horizontal standard GPS coordinates, and the differential GPS coordinates. In road sections where the model was used to determine the gradients, errors of the speedometer and the power meter measurements could cause errors in the resulting gradients, too. An a-posteriori re-computation of the gradient using the model and the measured values for power and speed of the first ride (Ottenberg, average gradient $8.3 \%$ ) yielded a modified gradient which, by construction, leads to model prediction errors that completely vanish for that ride. These modified gradients are on average $0.13 \%$ lower than the measured gradients, i.e., the mean modified gradient was $8.17 \%$. The standard deviation of the difference between the computed and the measured gradients was $0.81 \%$. Assuming a sampling distance of approximately $3 \mathrm{~m}$, the standard deviation of $0.81 \%$ of the measured gradient values corresponds to about $2.4 \mathrm{~cm}$ deviation in height difference measurements between two adjacent sample points. This is in the range of the standard deviation estimated by the Leica GPS900 for good signal reception. Therefore, this rough and simplifying analysis indicates that the observed model prediction errors are approximately in the range that can be expected due to measurement errors in differential GPS altitude.

\subsection{Comparison of simulator measurements with model predictions}

The measurements of power and speed on the simulator agreed very well with the mathematical model predictions with a signal-to-noise-ratio ranging from $23.2-27.4 \mathrm{~dB}$, see Figure 5. In contrast to the above the causes for artifacts cannot be explained by insufficiencies of the mathematical model since it is the model itself which was implemented in the simulator.

Apart from errors induced by the brake force generation and the speed measurement of the ergometer, small deviations were due to the different numerical implementation of the simulator and the model prediction. The simulation software has a fixed sampling rate of $2 \mathrm{~Hz}$ and future values of speed and distance for the computation of the gradient and the acceleration for the next sampling period are unavailable. In contrast, for the model predictions Matlab's ode45 function was used which includes the computation of variable step sizes based on accuracy estimations.

There were two more technical sources of error: Firstly, a change of the electronic gears causes a small jump of the speed according to (9), which does not occur when shifting real gears. If power is computed by the model using the speed measurement, this speed jump represents an acceleration that will produce a corresponding short term power due to the change of the kinetic energy of the system. Secondly, when the minimal nominal pedal force fell below the $50 \mathrm{~N}$ threshold, as described in Section 1.3, the simulator 
switched to its model mode in which the speed was solely computed by the model and decoupled of the physical rotation speed of the ergometer. Hence, switching between normal and model mode again caused a speed jump and a corresponding power prediction. However, with the setup of the rides presented in this paper, these situations were rare. Such errors can been seen in road sections where the model mode is active, i.e., in the first ride at $2.55-2.60 \mathrm{~km}$ and in the second ride at $1.82-1.94 \mathrm{~km}$ and $4.04-4.13 \mathrm{~km}$.

The density functions of the error were again hat-shaped with values for the mean and the standard deviation less than with the comparison of model prediction and SRM field measurements. The accuracy of the power measurements after ergometer calibration by the manufacturer is claimed to be $\pm 2 \%$ for a power larger than $100 \mathrm{~W}$, [15]. Related to the average power of the first ride, this is equivalent to $\pm 4.1 \mathrm{~W}$ and could already explain a substantial part of the error.

\subsection{Comparison of simulated rides with outdoor rides}

The (unnormalised) performance parameters in the simulations were only partly similar to those in the field, with a range of 14.1-19.6 dB in SNR. The curve in Figure 6 shows the best result (cyclist A) only. As in the previous figures, quality measures to compare the similarity of outdoor and simulator ride are given for all rides in the table below.

However, part of the deviations were caused by the inability of the cyclists to cycle with exactly the same speed/power in the field and on the simulator, see Figure 7. The extent of this contribution to the overall error depends on the cyclist and may decrease with experience. Moreover, it is irrelevant for the research question on hand to ask to what precision a lab simulation can reproduce real-world conditions for a given track in the field. In order to compensate for this irrelevant error, the normalisation, as proposed in Section 2.6, was applied and yielded the comparison depicted in Figure 8.

The signal-to-noise ratios of $19.5-24.6 \mathrm{~dB}$ and all other error measures for the normalised measurements were very similar to those of the comparison of model predictions and SRM field measurements. This is not surprising because the model prediction was much closer to the simulator measurements than to the SRM field measurements.

However, a further source of error must be considered when power measured by the simulator is compared to power measured by the SRM: Although both measurement devices were calibrated by the manufacturers within the recommended time period of one year, the power measured by the SRM during cycling on the ergometer is only $95 \%$ of the power measured by the ergometer in the range between $100-300 \mathrm{~W}$. In fact, one should expect it to be even larger because the chain losses are only measured by the SRM in the crank and not by the simulator in the rear hub. This bias was the result of a systematic analysis of a step test in [11] where the same measurement devices were used. Upon request, the manufacturers confirmed their awareness of this problem. There is no perfect reference or ground truth for pedalling power and a scientific evaluation of different calibration techniques - either static using weights or dynamic using electrical generators or torque and angular speed measurements has not been done, yet.

\section{Conclusions and future work}

This study confirmed that a mathematical model for performance parameters in road cycling is capable of accurately predicting required power output also on uphill courses with variable road gradients given the location and speed along with physical, mechanical, and geographical parameters. Alternatively, the model can accurately predict the speed of the cyclist given the power applied at the chain wheel.

The acquisition of precise gradient profiles of the courses was a decisive factor for the accuracy of the prediction. In a similar previous study with slope profiles that originated from finite difference approximations of standard GPS altitude measurements using the Garmin Edge 705 [17], it was necessary to smooth the slope profile with a Gaussian filter of $30 \mathrm{~m}$ standard deviation in order to reduce spurious gradient peaks caused by noise. However, the smoothing decreased the magnitudes of narrow maximums and minimums, too. This led to visibly smaller model predictions of power at real gradient maximums and larger predictions at minimums. Here, this source of error was eliminated by replacing the smoothed height profiles by differential GPS and model predictions together with spatial referencing by means of GPS coordinates. However, the degraded quality or unavailability of the differential GPS signal in presence of trees, houses, and hills, as shown by the grey sections in Figure 1, was a significant limitation. The alternative method that computes the slope profile with the model equations, as described in Section 2.4, gave better slope profiles than with smoothed standard GPS data. Neither in the slope profile in Figure 1 nor in the comparison of model prediction and measurements, a significant degradation of the slope profile on course sections, where differential GPS is unavailable, could be detected visually. However, a drift error regarding the absolute height computed from this slope estimate could only be avoided if the slope is slightly scaled to match the true height differences of the start and end points of the sections.

It was shown that for simulated rides on real courses the mathematical model can be implemented on an ergometer which allows a fast and precise control of the brake force like the Cyclus 2. The simulation was accurate to a very good extent, so that the major contribution of errors between 
performance measurement on the simulator and outdoors was caused by the uncertainties of the model parameters and slope measurements and not by the ergometer control. This is true provided that the simulated resistance is in the valid (positive) range depending on the angular speed of the ergometer. Therefore, the simulator can be used for course specific training. A comparison of a field and simulator ride requires to consider the deviation induced by the inability of a cyclist to maintain the same pacing during the two rides.

This study had some limitations; it incorporated (also steep) slopes, but not high speeds, which would test for accuracy of the model regarding higher order terms of the speed. However, this was the focus in another study [11] where the use of the model to estimate the physical parameters like the rolling friction coefficient or the cross-sectional area of the cyclist-bicycle system was dealt with. Those experiments were performed on a flat road so that high speeds could be reached. A comparison with literature parameters was included.

Another limitation was that downhill sections have not been considered, yet. Although, for downhill cycling, the simulator is able to compute the kinetic variables according to the model and play the video with the appropriate speed, there is no motor that could accelerate the ergometer mechanics. Besides, downhill cycling requires dexterity rather than physical power capabilities and endurance, the latter of which is the main focus of this research project. The necessity of braking when speed is too high or, e.g., balancing on the bicycle in a curve is difficult to model and simulate and cannot be trained on ergometers of current technology.

Future work will improve the operation of the simulator by controlling the brake at a $10 \mathrm{~Hz}$ instead of $2 \mathrm{~Hz}$ sampling rate using the RS232 interface. Thus, the mismatch of the moment of inertia can be compensated by incorporating an appropriate term into the brake force and a smoother transition between normal and model mode in presence of downhill sections can be designed.

Although in principle the current road gradient measurements are accurate enough, the Galileo GNSS, which is currently being developed, may provide a better accuracy and, more importantly, better availability of accurate position data also in road sections with obstacles nearby. If even bicycle computers get equipped with that new technology, gradient profiles of further courses will be measurable with a simpler and more practical technique and sufficient precision.

Both the model and the simulator have been used to compute and validate the optimum pacing strategy for individual cyclists which requires a physiological model of the physical power capabilities of the cyclist [9]. This approach was motivated and related to previous work by Maronski, 1994 [8], Gordon, 2005 [7], and Atkinson, 2007 [6]. Together with an extension of physiological measurements and their modelling the whole system shall indicate and train effective tactics enabling cyclists to optimally prepare even for unfamiliar tracks.

Acknowledgements We thank Dr. Dietmar Lüchtenberg of the Department of Sport Science of the University of Konstanz and the anonymous reviewer for discussions and support. A part of this work was carried out while the third author was a Visiting Research Fellow at RSISE, Australian National University, Canberra, whose support is gratefully acknowledged.

\section{References}

1. di Prampero, P.E., Cortili, G., Mognoni, P., Saibene, F.: Equation of motion of a cyclist. Journal of Applied Physiology 47(1), 201206 (1979)

2. Martin, J.C., Milliken, D.L., Cobb, J.E., McFadden, K.L., Coggan, A.R.: Validation of a mathematical model for road cycling power. Journal of Applied Biomechanics 14, 276-291 (1998)

3. Olds, T.S., Norton, K.I., Craig, N.P.: Mathematical model of cycling performance. Journal of Applied Physiology 75(2), 730-737 (1993)

4. Olds, T.S., Norton, K.I., Lowe, E.L., Olive, S., Reay, F., Ly, S.: Modeling road-cycling performance. Journal of Applied Physiology 78(4), 1596-1611 (1995)

5. Atkinson, G., Brunskill, A.: Pacing strategies during a cycling time trial with simulated headwinds and tailwinds. Ergonomics 43(10), 1449-1460 (2000)

6. Atkinson, G., Peacock, O., Passfield, L.: Variable versus constant power strategies during cycling time-trials: Prediction of time savings using an up-to-date mathematical model. Journal of Sports Sciences 25(9), 1001-1009 (2007)

7. Gordon, S.: Optimising distribution of power during a cycling time trial. Sports Engineering 8(2), 81-90 (2005)

8. Maronski, R.: On optimal velocity during cycling. Journal of Biomechanics 27(2), 205-213 (1994)

9. Wolf, S., Dahmen, T.: Optimierung der Geschwindigkeitssteuerung während Zeitfahrten im Radsport. In D. Link, J. Wiemeyer (Eds.), Sportinformatik trifft Sporttechnologie, 8. Symposium der dvs-Sektion Sportinformatik, September, 15-17, Darmstadt, 235-239 (2010)

10. Lim, A.C., Homestead, E.P., Edwards, A.G., Carver, T.C., Kram, R., Byrnes, W.C.: Measuring changes in aerodynamic/rolling resistances by cycle-mounted power meters. Medicine \& Science in Sports \& Exercise 43(5), 853 (2011)

11. Dahmen, T., Saupe, D.: Calibration of a power-velocity-model for road cycling using real power and height data. International Journal of Computer Science in Sport, 2011, IACSS Press, to appear

12. Schoberer, E.: Operating instructions for the srm training system (1994). URL www.srm.de/index.php/de/support/phoca-downtest/category/14-powermeter (date accessed: Aug, 22th, 2011)

13. Gressmann, M.: Fahrradphysik und Biomechanik. Technik Formeln - Gesetze, 7. a. edn. Delius Klasing Verlag (2002)

14. Wilson, D., Papadopoulos, J., Whitt, F.: Bicycling science, 3 edn. The MIT Press (2004)

15. Cyclus2 website. URL www.cyclus2.com/en/index.htm, date accessed: Aug, 10th, 2011

16. Saupe, D., Lüchtenberg, D., Röder, M., Federolf, Ch.: Analysis and visualization of space-time Variant parameters in endurance sports training. Proceedings of the 6th International Symposium on Computer Science in Sports, IACSS (2007)

17. Dahmen, T., Byshko, R., Saupe, D., Röder, M., Mantler, S.: Modeling, simulation and validation of cycling time trials on real tracks. Konstanz Online Publication System. Retrieved from http://nbn-resolving.de/urn:nbn:de:bsz:352-133144 